\title{
Effect of Salinity and pH on Fatty Acid Profile of The Green Algae Tetraselmis suecica
}

Adel Waslallah Almutairi ${ }^{1}$ and Hussein Elsayed Toulibah ${ }^{1,2 *}$

${ }^{1}$ Biological Sciences Department, Rabigh-Faculty of Science and Arts, King Abdulaziz University, P. O. box 344, Rabigh 21911, Saudi Arabia

${ }^{2}$ Botany Department, College of Women for Arts, Science and Education - Ain Shams University - 1, Asmaa Fahmy Street - Heliopolis, Post cod No. 11757 - Cairo, Egypt

\begin{abstract}
In this study, the marine alga Tetraselmis suecica was cultured in F/2 medium with various rang of salinity and $\mathrm{pH}$. The influence of stressed algal cells to produce biofuels was studied. The neutral lipid (triacylglycerol) assembled by $T$. suecica was determined using Nile Red dye under salinity stress (up to $1 \mathrm{M} \mathrm{NaCl}$ ) and $\mathrm{pH}(\mathrm{pH} 7$ to 9 ). Salinity showed a stimulating effect on triacylglycerol in the algal cells at the 1 Molars, while the effect of $\mathrm{pH}$ was changeable. Then fatty acid profile of $T$. suecica cells was evaluated by gas chromatography-mass spectrometry (GC-MS) after direct trans-esterification with hydrochloric acid in methanol. It was shown that, high salinity was ideal for biodiesel production due to increasing of monounsaturated fatty acids produced by Tetraselmis suecica.
\end{abstract}

Keywords: Tetraselmis suecica; Neutral lipid (triacylglycerol); Salinity; $\mathrm{pH}$

\section{Introduction}

The majority of the energy request is met from the consuming of non-renewable energy sources (oil, petroleum gas and coal) which are simple to utilize, give high energy and are economy when compared other energy sources. The ability and response of Tetraselmis suecica in a batch culture with wide range of light regimes, nutrient concentrations and salinity were studied by Şen, Abmohra [1,2]. Hook and Tang [3] stated that, the continues using of fossil fuels are not recommended due to increase the air pollution as well as increase of rate the gathering of greenhouse gases (GHG) that lead to production of acid rain. In addition to the rapid depletion the fossil fuels resources. Therefore, a large amount of research has been carried out with the aim of finding new renewable energy sources that are sustainable and environmentally friendly. Christi [4], mentioned that using microalgae is more likely to produce higher levels of neutral lipids (triacylglycerol, TAG). The vital point to produce algal biodiesel in commercial scale is to find a strain with high production levels of neutral lipid. Fakhry and El Maghraby [5] studied the stress conditions by the nitrogen Depilation and variation of temperature on the growth of Nannochloropsis salina. Monika [6] studied the effect of high salinity, light intensity, photoperiod and $\mathrm{pH}$ on the growth and lipid accumulation of Chlorella sp. Miranda [7] studied the effect of nitrate, phosphate and salinity to enhance the accumulation of fatty acids Ankistrodesmus sp. and Chlamydomonas sp. The microalgal growth and metabolism will be affected by the change of some physic-chemical parameters as light, nutrients, temperature, salinity and $\mathrm{pH}[8]$. Miranda [6] discussed the energy transfer and biosynthesis of macromolecules bound in algal membrane were dependent on lipid composition, Hence, at any environmental stress the microalgae, the physiological function of the microalgae membrane tries to readjust to tolerate severe stress. This lead to, the microalgae accumulated the lipds in the form of triacylglycerol (TAG) up to $20 \%$ to $50 \%$ of their dry cell weight.

Algal lipids can be divided into polar and non-polar lipids. The biodiesel production come mainly from the non-polar lipid fraction, fatty acid attached with glycerol backbone (neutral lipid, triacylglycerol, TAG). Therefore, the suitability of microalgae for the production of biodiesel depends on their TAG content. Consequently, it must be understanding at what growth stage and under which culture conditions that TAG accumulation is maximised. Since TAG is synthesized as a storage compound, it makes sense to stress the algae using environmental factors like temperature, light intensity, $\mathrm{pH}$ and salinity to reduce growth and switch on TAG production by altering the activity of metabolic pathways [9]. However, this means that growth is halted to induce TAG synthesis, rather than having TAG synthesized as the cells grow. The latter is more desirable since it would lead to higher productivity. The other aspect of TAG synthesis is the composition of the fatty acid chains. The number of characteristics of the fatty acids can change including length (number of carbons) and presence/absence and number of double bonds (i.e., degree of unsaturation). The properties of the fatty acids affect the properties of the biodiesel produced [10].

The composition of diesel fuels is covered by strict standards throughout the world (e.g. EN 14214 is the fuel standard used in Europe). This standard ensures that the ignition properties of the fuel, its viscosity and oxidative stability all fall within acceptable limits [10]. Fully saturated fatty acids tend to add stability to the fuel, but can cause problems in cold weather [11]. Fatty acids rich in monounsaturated fatty acids are likely to be suitable and should make up a high proportion of the biodiesel. Polyunsaturated fatty acids are detrimental to fuel due to their susceptibility to oxidation [10]. The chain length is also important with $\mathrm{C} 16$ and $\mathrm{C} 18$ fatty acids being most suitable. Prakasam discussed the algal biomass, lipid and carbohydrate production of Nannochloropsis salina, Dunaliella tertiolecta and Tetraselmis suecica as a result of stressing the F2/medium by different carbon sources. Abomohra [2] discussed the efficiency of Tetraselmis elliptica isolated from Bardawil lagoon (hypersaline water) as a hopeful species for biodiesel feedstock. The maximum production of monounsaturated fatty acid profile of $T$. elliptica was recorded at the late exponential phase of the growth curve.

"Corresponding author: Toulibah HE, Biological Sciences Department, RabighFaculty of Science and Arts, King Abdulaziz University, P. O. box 344, Rabigh 21911, Saudi Arabia, Tel: +966555878142; E-mail: touliabah2@hotmail.com

Received May 25, 2017; Accepted June 22, 2017; Published June 28, 2017

Citation: Almutairi AW, Toulibah HE (2017) Effect of Salinity and pH on Fatty Acid Profile of The Green Algae Tetraselmis suecica. J Pet Environ Biotechnol 7: 333. doi: $10.4172 / 2157-7463.1000333$

Copyright: ( 2017 Almutairi AW, et al. This is an open-access article distributed under the terms of the Creative Commons Attribution License, which permits unrestricted use, distribution, and reproduction in any medium, provided the original author and source are credited. 
Current study focused the enhancement correlation between increased salinity in the medium as well as $\mathrm{pH}$ and the production of neutral lipids (TAG) of the T. suecica.

\section{Materials and Methods}

\section{Microalgae and growth medium}

Tetraselmis suecica (CCAP 66/4) was obtained from the Culture Collection of Algae and Protozoa, Oban, UK [12]. A primary stock culture (in a $100 \mathrm{ml}$ flask) was prepared using the liquid T. suecica samples received from the CCAP and allowed to grow in the culture room at $25 \pm 1^{\circ} \mathrm{C}$ with continuous light $\left(50-70 \mu \mathrm{mol} \mathrm{m} \mathrm{m}^{-2} \mathrm{~s}^{-1}\right)$ supplied by daylight fluorescent lights. When the culture reached the stationary phase after around 2 weeks incubation, F/2 was inoculated from the primary stock culture and allowed to grow to allow for adaptation to the medium before starting experiment.

\section{F/2 medium}

$\mathrm{F} / 2$ Medium is an artificial seawater medium and was prepared as described in Guillard and Ryther [13].

\section{Lipid determination by Nile red fluorescence}

A number of experiments have been done to measure neutral lipid concentration in T. suecica. Firstly, droplets of neutral lipids were visualised using a fluorescence microscope. Secondly, optimisation of Nile Red Fluorescence emission using the 96 well microplate methods involved optimisation of Nile Red concentration, cell concentration and time of staining.

Lipid body visualisation using a fluorescence microscope: Qualitative analyses of microalgae accumulate and store natural lipids in lipid bodies which appear as oil droplets inside their body. For visualisation of those lipid bodies Nile Red lipo-philic dye was used for staining and a Fluorescence Microscope with a Nikon Digital camera attached was used to capture the images. This approach is based on the work by Cooksey [14].

Nile red sample measurement test: Nile Red measurements on algal cells grown under different environmental conditions were done after performing optimisation test for Nile Red peak fluorescence and Nile Red concentration test based on the work done by Alonzo, Chen and Bertozzini [15-17]. Also, to find the optimise time for harvesting cells during the induction of lipid accumulation, measurements were performed on cells grown for 1 week, 2 weeks, 3 weeks and 4 weeks.

Lipid determination by direct trans-esterification methods: This work was done based on methods demonstrated by Griffiths [18]. The extracted FAMEs from the transesterification reactions were then identified by gas chromatography mass spectroscopy (GC-MS) using a Perkin Elmer - Auto System XL Gas Chromatograph (CHM-100790) and Perkin Elmer - Turbo Mass Mass-Spectrometer (13657). The resultant peaks were identified and integrated using a Perkin Elmer's Turbo mass software linked to a NIST database.

\section{Results}

\section{Visualisation of lipid bodies using fluorescence microscopy}

The accumulation of neutral lipids in T. Suecica cells was first monitored by examining NR fluorescence under the fluorescence microscope to check that the NR dye dissolved in acetone was able to penetrate into the lipid droplets within the T. Suecica cells. The microscope images in Figure 1 clearly show the yellow fluorescence of
NR when it is staining neutral lipids (TAG). The staining shows that the neutral lipids are present as discrete droplets within the cells. There may be some indication that the amount of neutral lipid increased with increasing salinity, middle. There may be some indication that the amount of neutral lipid increased with increasing salinity, B) middle images being from highest salinity (Figure 1), but this is not a quantitative method. The key finding is that NR in acetone successfully entered the T. Suecica cells and that the stained neutral lipid droplets were obviously visualised under the fluorescence microscope.

Neutral lipid content of $T$. suecica cells grown at different salinities

This experiment focused to determine if there is any correlation between the increasing of salinity and the production of neutral lipids (TAG) in T. suecica. The accumulation of lipids in T. suecica was assessed using NR dye staining as optimised using $3 \mu \mathrm{mol} / \mathrm{ml}$ of NR dye concentration with the concentration of $T$. suecica cells set at $\mathrm{OD}_{595}=0.818(87.5 \%$ cell concentration $)$ and the measurement of fluorescence intensities $20 \mathrm{~min}$. after staining the algal cells with NR dye. The test was performed every week for four weeks to investigate the effect of salt stress over a significant time period. Figure 2 shows that the highest levels of neutral lipids were observed with the highest $\mathrm{NaCl}$ concentration $(1 \mathrm{M})$ after 4 weeks culture. The relationship between increasing the salt concentration and TAG accumulation was not straight forward. After two weeks growth, the lowest levels of TAG were found in the $1 \mathrm{M} \mathrm{NaCl}$ grown cells (Figure 2). It is clear that, age of culture is also important when considering the optimum conditions for TAG accumulation.

\section{Neutral lipid content of $T$. suecica grown at different ph values}

The influence of $\mathrm{pH}$ on the neutral lipid content of T. suecica was examined using NR staining dye as optimised using $3 \mu \mathrm{mol} / \mathrm{ml}$

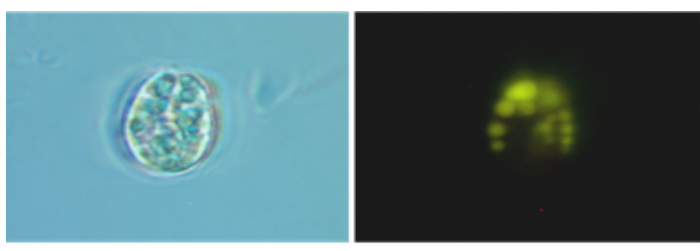

a) Cells grown in $0.4 \mathrm{M} \mathrm{NaCl} \mathrm{F/2} \mathrm{medium.}$

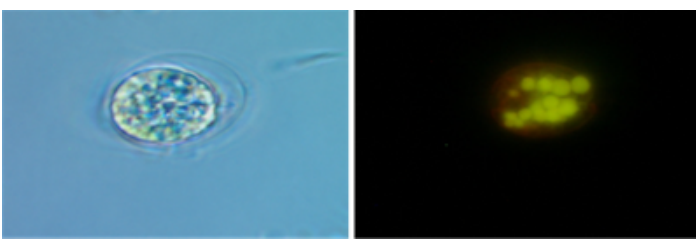

b) Cells grown in $1 \mathrm{M} \mathrm{NaCl} \mathrm{F/2} \mathrm{medium.}$

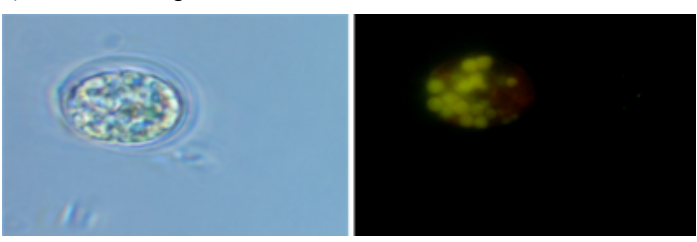

c)

Cells grown in $0.6 \mathrm{M} \mathrm{NaCl} \mathrm{F/2} \mathrm{medium.}$

Note: The images on the left are taken under normal light and the images on the right show the same cell under fluorescent light conditions. The key finding is that NR in acetone successfully entered the T. Suecica cells and that the stained neutral lipid droplets were clearly visualised under the fluorescence microscope.

Figure 1: Fluorescence microscopy images of $T$. suecica cells stained with NR at a final concentration of $1 \mu \mathrm{mol} / \mathrm{ml}$ 
concentration of NR dye with the concentration of T. suecica cells $\left(\mathrm{OD}_{595}=0.818\right)$ and the measurement of fluorescence intensities at 20 min. after staining the algal cells with NR dye. The test was performed each week from 1 to 4 weeks after inoculation to examine the effect of $\mathrm{pH}$ stress over a long period. Figure 3 shows that, interestingly, the highest neutral lipid levels were observed in T. suecica cells grown at $\mathrm{pH} 9$ for 2 weeks.

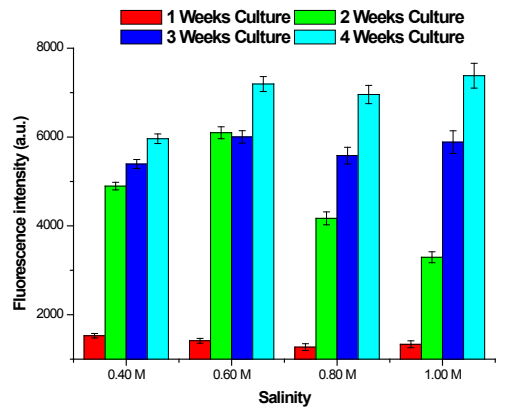

Figure 2: Neutral lipid content measured by NR fluorescence of $T$. suecica in F/2 medium with different concentrations of $\mathrm{NaCl}$.

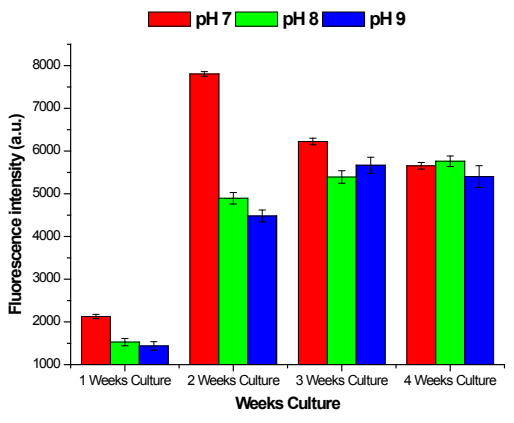

Figure 3: Effect of $\mathrm{pH}$ on Neutral lipid content measured by NR fluorescence of $T$. suecica cells grown in $\mathrm{F} / 2$ medium at different $\mathrm{pH}$ values.
From our result, by increasing $\mathrm{pH}$ values the neutral lipid values showed a remarkable increase till the fourth week of the experiments. Then the increase in $\mathrm{pH}$ values was not effective on the neutral lipid values anymore. The effects of $\mathrm{pH}$ are presented in Figure 3.

\section{Fatty acid profiles of $T$. Suecica cells grown at different} salinities

Figures 4-6 show the spectra of fatty acids found in T. suecica cells exposed to increasing salinity. In each figure, the top panel shows the fatty acid spectrum for cells grown in normal salinity $(0.4 \mathrm{M} \mathrm{NaCl})$ and two saturated fatty acids dominate the spectrum - hexadecanoic acid (palmitic acid, C16:0) and octadecanoic acid (stearic acid, C18:0). In cells grown in $0.6 \mathrm{M} \mathrm{NaCl}$, several other fatty acids are detected in addition to the two-major saturated fatty acids i.e., pentadecanoic acid (C15:0), cis-10-heptadecanoic acid (C17:1), elaidic acid (C18:1) and linolelaidic acid (C18:2). At higher salinities (0.8 and $1 \mathrm{M} \mathrm{NaCl})$, the same spectrum of additional fatty acids were found (Figures 5 and 6) It seems clear that with increased salinity above $0.4 \mathrm{M} \mathrm{NaCl}$, more unsaturated fatty acids are produced in response to the salt stress.

\section{Fatty acid profiles of $T$. suecica grown at different $\mathrm{pH}$ values}

Figures 7 and 8 shows that stressing the T. suecica cells, through changing the external $\mathrm{pH}$ to 7 or 9 , had a very similar effect on the fatty acid profile as increasing the salinity. Again, a number of unsaturated fatty acids were produced in response to the change in $\mathrm{pH}$ including linolelaidic acid, which contains two double bonds and is a polyunsaturated fatty acid.

\section{Discussion}

The work aimed to identify the best conditions for neutral lipid accumulation in Tetraselmis suecica. This alga has been identified as a good candidate for the production of biodiesel and other fine chemicals on the basis of high lipid content and the fact that T. suecica is tolerant to a range of environmental extremes including high salinity and $\mathrm{pH}$ [19].

Nile Red (NR) fluorescence was shown to be a right method for
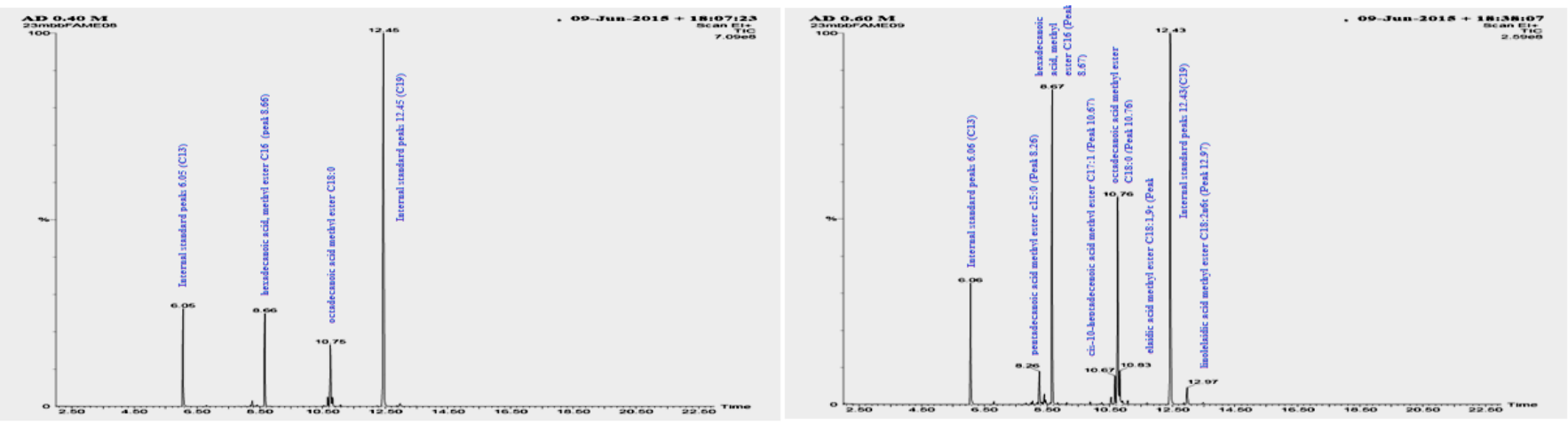

4a: $T$. suecica grown in normal conditions with $0.4 \mathrm{M}$ salt concentration and $\mathrm{pH} 8$.

The fatty acids accumulated were identified as:

(1) - hexadecanoic acid, methyl ester C16 (peak 8.66)

(2) - octadecanoic acid methyl ester C18:0 (Peak 10.75) and the other two peaks were internal standard peaks 6.05 (C13) and peak 12.45 (C19).

4b: T. Suecica cells grown under stress conditions with $0.6 \mathrm{M}$ salt concentration and the fatty acids accumulated were:

(1) Pentadecanoic acid methyl ester c15:0 (Peak 8.26),

(2) Hexadecanoic acid, methyl ester C16 (Peak 8.67),

(3) Cis-10-heptadecenoic acid methyl ester C17:1 (Peak 10.67),

(4) Octadecanoic acid methyl ester C18:0 (Peak 10.76),

(5) Elaidic acid methyl ester C18:1,9t (Peak 10.83),

(6) Linolelaidic acid methyl ester C18:2n6t (Peak 12.97).

Figure 4: Comparison of GC-MS chromatographs for fatty acid accumulation in T. Suecica. 


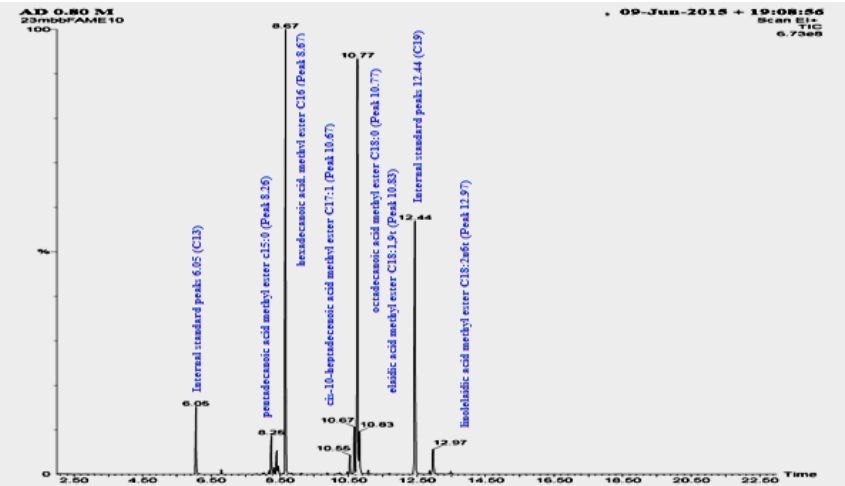

$T$ suecica cells grown under stress conditions with $0.8 \mathrm{M}$ salt concentration and the fatty acids accumulated were:

(1) Pentadecanoic acid methyl ester c15:0 (Peak 8.26)

(2) Hexadecanoic acid, methyl ester C16 (Peak 8.67),

(3) Cis-10-heptadecenoic acid methyl ester C17:1 (Peak 10.67),

(4) Octadecanoic acid methyl ester C18:0 (Peak 10.77),

(5) Elaidic acid methyl ester C18:1,9t (Peak 10.83),

(6) Linolelaidic acid methyl ester C18:2n6t (Peak 12.97).

The other two peaks were internal standard peaks 6.05 (C13) and 12.44 (C19).

Figure 5: Comparison of GC-MS chromatographs for fatty acid accumulation in T. suecica.

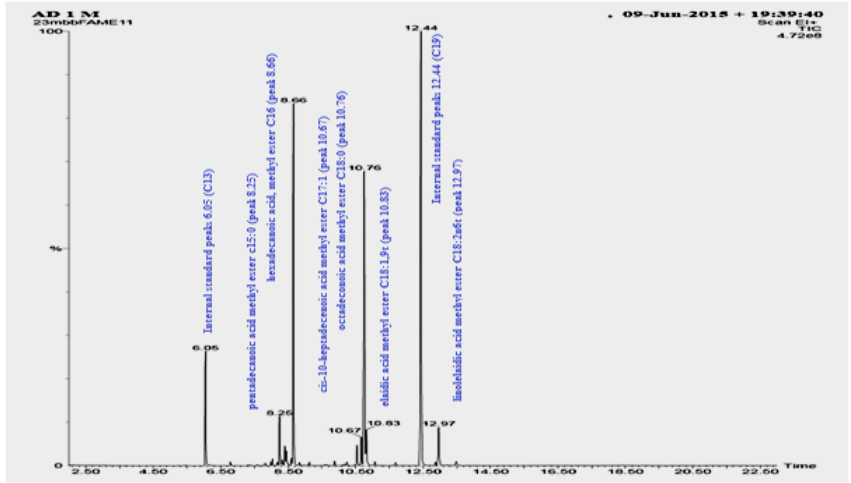

T. suecica grown under stress conditions with $1 \mathrm{M} \mathrm{NaCl}$ concentration and the fatty acids accumulated were:

(1) Pentadecanoic acid methyl ester c15:0 (peak 8.25)

(2) Hexadecanoic acid, methyl ester C16 (peak 8.66),

(3) Cis-10-heptadecenoic acid methyl ester C17:1 (peak 10.67),

(4) Octadeconoic acid methyl ester C18:0 (peak 10.76),

(5) Elaidic acid methyl ester C18:1,9t (peak 10.83),

(6) Linolelaidic acid methyl ester C18:2n6t (peak 12.97).

The other two peaks were internal standard peaks 6.05 (C13) and 12.44 (C19).

Figure 6: Comparison of GC-MS chromatographs for fatty acid accumulation in T. Suecica.

visualising lipid droplets inside T. suecica cells (Figure 1). The NR fluorescence method was then optimised for a quantitative assay in a 96 well plate reader format by setting the time required for peak fluorescence and the concentration of both cells and NR dye that are optimal. Using these parameters, the amount of neutral lipid (TAG) accumulated by $T$. suecica cells under salinity and $\mathrm{pH}$ stress was measured (Figure 2 and 3 ).

At the normal $\mathrm{pH}$ of 8 , it was found that four weeks incubation at 1 $\mathrm{M} \mathrm{NaCl}$ gave rise to the highest amount of TAG (Figure 2). In contrast at the normal salinity of $0.4 \mathrm{M} \mathrm{NaCl}$, it was found that 2 weeks culture at $\mathrm{pH} 9$ produced the most TAG. This established that environmental stresses can turn on TAG synthesis as has been shown previously for N-limited Tetraselmis cultures [20].

\section{Conclusion}

The conclusion to be drawn is that a large increase in salinity can induce increased TAG accumulation and high salinity may be the best condition to use for TAG synthesis in a commercial biofuel process based on T. suecica as the feedstock. This has the additional advantage that contamination of T. suecica cultures will be more easily controlled at elevated salinities [21-24].

The fatty acid profiles of the TAG accumulated under salinity and $\mathrm{pH}$ stresses were measured using GC-MS. Any increase in salinity above $0.4 \mathrm{M} \mathrm{NaCl}$ induced the production of monounsaturated fatty acids and one polyunsaturated fatty acid. The mixture of saturated, unsaturated and polyunsaturated fatty acids looks very promising as a base for biodiesel production and should meet the requirements for cold-flow, ignition properties, viscosity and oxidative stability [10]. The same behaviour of Nannochloropsis saline was be obtained by Fakhry and El Maghraby [5]. They attributed the maximum production of the lipids as biodiesel feedstock was obtained as a result of depletion of 
Citation: Almutairi AW, Toulibah HE (2017) Effect of Salinity and pH on Fatty Acid Profile of The Green Algae Tetraselmis suecica. J Pet Environ Biotechnol 7: 333. doi: 10.4172/2157-7463.1000333

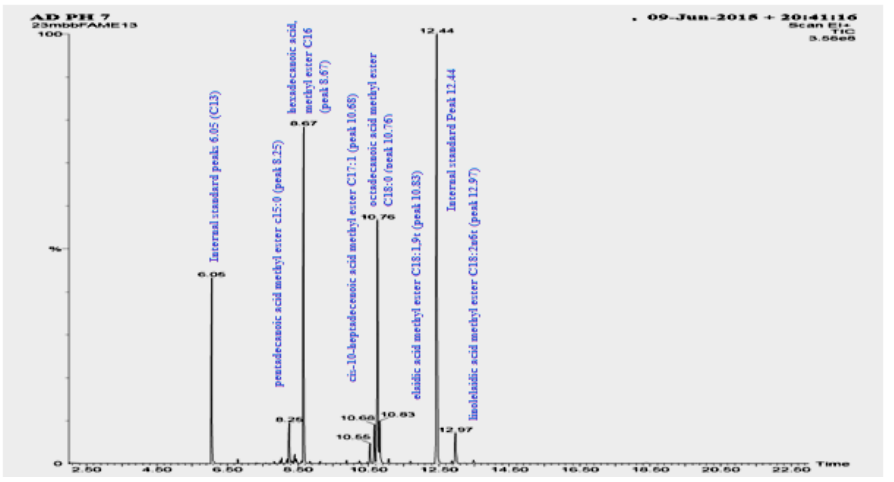

T. suecica grown under stress conditions at $\mathrm{pH} 7$ and the fatty acids accumulated were:

(1) Pentadecanoic acid methyl ester c15:0 (peak 8.25)

(2) Hexadecanoic acid, methyl ester C16 (peak 8.67),

(3) Cis-10-heptadecenoic acid methyl ester C17:1 (peak 10.68),

(4) Octadecanoic acid methyl ester C18:0 (peak 10.76),

(5) Elaidic acid methyl ester C18:1,9t (peak 10.83)

(6) Linolelaidic acid methyl ester C18:2n6t (peak 12.97).

The other two peaks were internal standard peaks 6.05 (C13) and Peak 12.44 (C19).

Figure 7: Comparison of GC-MS chromatographs for fatty acid accumulation in T. Suecica.

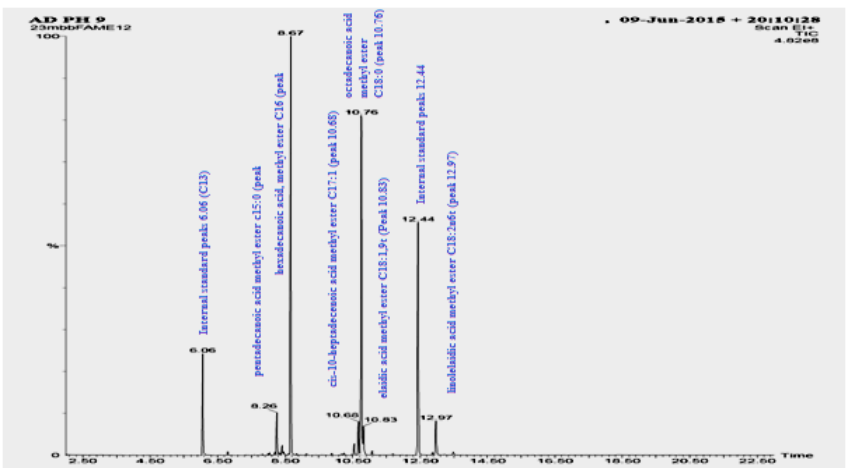

$T$. suecica grown under stress conditions at $\mathrm{pH} 9$ and the fatty acids accumulated were:

(1) Pentadecanoic acid methyl ester c15:0 (peak 8.26)

(2) Hexadecanoic acid, methyl ester C16 (peak 8.67).

(3) cis-10-heptadecenoic acid methyl ester C17:1 (peak 10.68)

(4) Octadecanoic acid methyl ester C18:0 (peak 10.76),

(5) Elaidic acid methyl ester C18:1,9t (Peak 10.83),

(6) Linolelaidic acid methyl ester C18:2n6t (peak 12.97).

The other two peaks were internal standard peaks 6.06 (C13) and 12.44 (C19).

Figure 8: Comparison of GC-MS chromatographs for fatty acid accumulation in T. Suecica.

nitrogen and high levels of temperature from Nannochloropsis saline [25-30].

The fatty acid profiles were also determined using GC-MS and increasing salinity induced the synthesis of fatty acids with one double bond (monounsaturated), which are ideal for biodiesel production $[10,11]$. It appears likely that growing $T$. suecica at high salinity will result in a favourable mix of fatty acids for biodiesel.

\section{References}

1. Şen B, Koçer MAT, Alp MT (2005) Studies on growth of marine microalage in batch cultures: IV. Tetraselmis suecica (Kylin) Butcher (Prasinophyta). AJPS 4: 645-647.

2. Abomohra A, El-Sheekh MM, Dieter H (2017) Screening of marine microalgae isolated from the hypersaline Bardawil lagoon for biodiesel feedstock. Renewable Energy 101: 1266e-1272.
3. Hook M, Tang X (2013) Depletion of fossil fuels and anthropogenic climate change - A review. Energ. Policy 52: 797-809.

4. Christi $Y$ (2008) Biodiesel from microalgae beats bioethanol. Trends Biotechnol 26: $126-131$.

5. Fakhry EM, El Maghraby DM (2015) Lipid accumulation in response to nitrogen limitation and variation of temperature in Nannochloropsis salina. Botanical Studies 56: 6 .

6. Monika PR, Trishnamoni G, Nikunj S (2015) Effect of Salinity, pH, Light Intensity on Growth and Lipid Production of Microalgae for Bioenergy Application. J Biol Sci 15: 260-267.

7. Miranda CT, de Lima DVN, Atella GC, de Aguiar PF, Azevedo SMFO (2016) Optimization of nitrogen, Phosphorus and salt for lipid accumulation of microalgae: Towards the viability of microalgae Biodiesel. Natural Science 8 : 557-573.

8. Sommer U (1999) A comment on the proper use of nutrient ratios in microalga ecology. Archiv fur Hydrobiologie 146: 55-64. 
Citation: Almutairi AW, Toulibah HE (2017) Effect of Salinity and pH on Fatty Acid Profile of The Green Algae Tetraselmis suecica. J Pet Environ Biotechnol 7: 333. doi: 10.4172/2157-7463.1000333

Page 6 of 6

9. Huang XX, Huang ZZ, Wen W (2013) Effects of nitrogen supplementation of the culture medium on the growth, total lipid content and fatty acid profiles of three microalgae (Tetraselmis subcordiformis, Nannochloropsis oculata and Pavlova viridis). J. Appl. Phycol. 25: 129-137.

10. Stansell GR, Gray VM, Sym SD (2012) Microalgal fatty acid composition implications for biodiesel quality. J. Appl. Phycol. 24: 791-801.

11. Knothe G (2005) Dependence of biodiesel fuel properties on the structure of fatty acid alkyl esters. Fuel Process. Technol. 86: 1059-1070.

12. Butcher RW (1959) An introductory account of the smaller algae of British coastal waters. Part I: Introduction and Chlorophyceae. Fisheries Investigations, London, series IV 1: 1-74

13. Guillard R, Ryther J (1962) Studies of marine planktonic diatoms. I. Cyclotella nana Hustedt and Detonula confervaceae (Cleve) Gran. Can. J. Microbiol. 8: 229-239.

14. Cooksey KE, Guckert JB, Williams SA, Callis PR (1987) Fluorometricdetermination of the neutral lipid content of microalgal cells using Nile red. $J$ Microbiol Methods 6: 333-345.

15. Alonzo F, Mayzaud P (1999) Spectrofluorometric quantification of neutral and polar lipids in zooplankton using Nile red. Mar. Chem. 67: 289-301.

16. Chen W, Zhang C, Song L, Sommerfeld M, Hu Q (2009) A high throughput Nile red method for quantitative measurement of neutral lipids in microalgae. J. Microbiol. Methods 77: 41-47.

17. Bertozzini E, Galluzzi L, Penna A, Magnani M (2011) Application of the standard addition method for the absolute quantification of neutral lipids in microalgae using Nile red. J Microbiol Methods 87: 17-23.

18. Griffiths MJ, Van Hille RP, Harrison STL (2010) Selection of direct transesterification as the preferred method for assay of fatty acid content of microalgae. Lipids 45: 1053-1060.

19. Chisti $Y$ (2007) Biodiesel from microalgae. Biotechnol. Adv. 25: 294-306

20. Xu D, Gao Z, Li F, Fan X, Zhang X, et al. (2013) detection and quantitation of lipid in the microalga Tetraselmis subcordiformis (Wille) Butcher with BODIPY 505/515 staining. Bioresour. Technol. 127: 386-390.
21. Azma M, Mohamad R, Rahim RA, Ariff AB (2010) Improved protocol for the preparation of Tetraselmis suecica axenic culture and adaptation to heterotrophic cultivation. The Open Biotechnol J. 4: 36-46.

22. Camacho RF, Garcia CF, Fernandez SJM, Chisti Y, Molina GE (2003) A mechanistic model of photosynthesis in microalgae. Biotechnol Bioeng. 81 459-473.

23. Hidalgo P, Toro C, Ciudad G, Navia R (2013) Advances in direct transesterification of microalgal biomass for biodiesel production. Rev. Environ Sci Biotechnol 12: 179-199.

24. Hughes SR, Gibbons WR, Moser BR, Rich JO (2013) Sustainable multipurpose biorefineries for third-generation biofuels and value-added co-products. Biofuels - Economy, Environ Sustain 10: 5772-54804.

25. Miranda CT, Pinto RF, De Lima DVN, Viegas CV, Costa SM, et al. (2015) Microalgae lipid and biodiesel production: A Brazilian challenge. AJPS 6: 2522 2533

26. Velu P, Peter MJ, Sanniyasi E (2015) Effect of various carbon sources on biochemical production in marine microalgae Nannochloropsis salina (Eustigmatophyceae), Dunaliella tertiolecta (Chlorophyceae) and Tetraselmis suecica (Chlorodendrophyceae). Int J Curr Microbiol App Sci 4: 207-215.

27. Qin JG, Pearson L, Carragher JF (1999) Response of Tetraselmis suecica to nutrient and grazer manipulation. J Appl Phycol 11: 481-485.

28. Reynolds CS (2006) The ecology of phytoplankton. Cambridge University Press, Cambridge, UK

29. Prakasam Velu, Maria Jenita Peter and Elumalai Sanniyasi (2015): Effect of Various Carbon Sources on Biochemical Production in Marine Microalgae Nannochloropsis salina (Eustigmatophyceae), Dunaliella tertiolecta (Chlorophyceae) and Tetraselmis suecica (Chlorodendrophyceae). Int. J. Curr. Microbiol. App. Sci (2015) 4(3): 207-215.

30. Minhas, A.K.M.; Peter, H.; Colin, J.B. and Alok, A. (2016): A review on the Assessment of Stress Conditions for Simultaneous Production of Microalgal Lipids and Carotenoids. Frontiers in Microbiology, Volume 7 | Article 54 